\title{
The effect of familiarity on the processing of fragmented figures
}

\author{
HOWARD S. HOCK \\ Florida Atlantic University, Boca Raton, Florida 33431 \\ and \\ NORMAN MARCUS \\ Johns Hopkins University, Baltimore, Maryland 21218
}

\begin{abstract}
The processing of fragmented figures was investigated in a same-different reaction time task with pairs of intact and pairs of fragmented alphabet letters. The effect of fragmentation was significantly larger for pairs of letters rotated into an unfamiliar orientation than for pairs of normally oriented letters. Since this significant interaction was obtained for subjects emphasizing a structural mode of processing (Hock, 1973), it was concluded that familiarity had a direct effect on the structural/organizational processing of the fragmented figures.
\end{abstract}

One of the most compelling phenomenological facts of perceptual psychology is the observation that a severely fragmented figure, though initially perceived as a disorganized jumble of pieces, is perceived as a well-organized whole when the figure is recognized. It is clear from this phenomenon that familiarity increases the likelihood that the fragmented pieces of a figure will be organized into a unitary whole. What remains uncertain is the locus of the familiarity effect.

In his classic study, Leeper (1935) found that verbal cues, such as superordinate category names, facilitated the recognition of fragmented figures. $\mathrm{He}$ also found that fragmented figures, which were initially recognized with great difficulty, were recognized immediately when viewed tachistoscopically several weeks later. Both these findings point to the importance of memory retrieval in reorganizing fragmented figures. Verbal cues can increase the likelihood that an appropriate memorial representation will be retrieved, and seeing a fragmented form for the second time can result in the retrieval of the "correct" reorganization that was achieved the first time the figure was seen. ${ }^{1}$ What remains unanswered, however, is whether the retrieved representation influences perceptual organization directly, or whether its influence occurs subsequent to organizational processes.

In interpreting his results, Leeper (1935) took the position that familiarity has a direct effect on perceptual organization. He argued that past experience introduces a perceptual organization that must

N. Marcus is currently at Stanford Medical School. The authors wish to thank Howard Egeth and Bert Green for their valuable contributions to this research. Reprint requests should be sent to the first author. successfully compete with spontaneous organizing forces if the subject is to recognize a fragmented figure. There are, however, other ways in which the memorial representation corresponding to a fragmented figure could have a direct influence on perceptual organization. For example, the memorial representation corresponding to a fragmented figure could serve as a schematic model that governs the organization of the pieces of the figure into a whole. This possibility would be consistent with Neisser's (1967) theory, which asserts that schematic information resulting from past experience can influence present acts of visual synthesis.

In contrast to the "direct" models described above, it is also possible that the effect of familiarity on the perceptual organization of fragmented figures is indirect. It is possible, for example, that the initial processing of a fragmented figure involves the generation of multiple tentative organizations of the fragments. Recognition would then result from the "selection" of the perceptual organization that is the best fit with a meaningful memorial representation. On this basis, familiarity would influence the ultimate perceptual organization of the fragmented figure, but its influence would take place at a stage of processing subsequent to the completion of organizational processes. Factors that influence memory retrieval (e.g., Leeper's verbal cues) would thereby affect the selection of a meaningful perceptual organization, but information in memory would not affect the act of organization itself. This kind of two-stage model of recognition is compatible with the traditional Gestalt position (Koffka, 1935; reaffirmed by Zuckerman \& Rock, 1957), which contends that past experience has no effect on organizational processes.

The purpose of the present study was to determine 
if familiarity has a direct effect on perceptual organization. The study was based on Sternberg's (1969) additive factors method for identifying stages of processing. According to this method, two variables are inferred to have affected the same stage of processing if they have an interactive effect on reaction time. If the two variables have an additive effect on reaction time, they are inferred to have affected different stages of processing.

In order to examine the stages of processing in the perception of fragmented figures, Leeper's procedure was simplified with respect to both the stimuli used and the task performed by the subject. The stimuli were varied with respect to fragmentation (intact vs. fragmented alphabet letters) and familiarity (alphabet letters presented in either their normal orientation or rotated $180 \mathrm{deg}$ into an unfamiliar orientation). The task involved the same-different comparison of pairs of intact and pairs of fragmented letters.

Two alternative models were considered. In the first it was proposed that familiarity and fragmentation affect the same stage of processing. This onestage model, which was consistent with the hypothesis that familiarity has a direct effect on organizational processes, would be supported by a significant interaction between the effects of familiarity and fragmentation. In the second model it was proposed that familiarity and fragmentation affect different stages of processing. This two-stage model was consistent with the hypothesis that the effect of familiarity occurs at a stage of processing subsequent to organizational processes. It would be supported by evidence that the effects of familiarity and fragmentation are additive.

Of further concern was the mode of processing underlying same-different comparison. When complex fragmented figures are used, as in Leeper's study, the increase in perceptual unity that accompanies recognition has sufficient phenomenal impact to clearly implicate organizational processing. In this simplified version of Leeper's task, it remained possible that "same" responses would not be based exclusively on organizational processes.

In a series of experiments, Hock (1973), Hock, Gordon, and Gold (1975), Hock, Gordon, and Marcus (1974), and Hock and Ross (1975) have obtained evidence for individual differences in the mode of processing underlying "same" comparison. These individual differences involve a distinction between structural and analytic modes of processing. Structural processes are hypothesized to involve the organization of the detailed parts of a stimulus into a well-formed whole. Analytic processes are hypothesized to involve the decomposition of stimulus information into a set of features.

Structural processes involve the use of Gestalttype organizational rules in conjunction with schem- atic information involving spatial relations. Thus, Hock and Ross (1975) inferred that subjects whose "same" responses were facilitated by the Gestalt property of symmetry had emphasized a structural mode of processing. For these subjects, it was found that familiarity facilitated the spatial rotation of one stimulus into the orientation of a second stimulus. In a subsequent study, Hock, Whitehurst, and Throckmorton (Note 1) also inferred that subjects whose "same" responses were facilitated by the Gestalt property of symmetry had emphasized a structural mode of processing. They found, for these subjects, that familiarity facilitated the spatial reproduction of dot patterns.

While structural processes involve referencing visual information to a spatial framework, analytic processes involve inferring the identity of a stimulus by attending to its spatially invariant distinctive features. Thus, Hock et al. (1975) inferred that subjects whose "same" reaction times were unaffected by the orientation of pairs of alphabet letters had emphasized an analytic mode of processing. It was found that these subjects based their "same" responses to pairs of physically identical letters on a comparison of verbal codes rather than on a more direct perceptual comparison. In a subsequent study involving word-picture comparison, emphasis on analytic processes was inferred for subjects whose reaction times were unaffected by the orientation of the pictures (Hock, Gordon, \& Corcoran, 1976). For these subjects, it was found that word-picture matches were faster when the words were general category names that when they were specific category names of the pictured object. Since more general levels of categorization require attention to fewer distinctive features, this result was consistent with Bruner's (1957) contention that categorization involves inferring the categorical identity of a stimulus from the minimal number of distinctive features.

The structural mode of processing hypothesized in the series of studies by Hock and associates and the organizational processes hypothesized by Leeper are comparable in their emphasis on the formation of perceptual wholes. The evidence for individual differences, however, suggesis that some subjects may process the stimuli analytically rather than structurally. That is, their "same" responses could be based on a comparison of distinctive features or verbal codes for the letters (Hock et al., 1975), and not a wholistic comparison of all the information in the fragmented letters.

Since the purpose of this study was to examine the effect of familiarity on the perceptual organization of the fragmented figures, the hypothesized stage models received their primary experimental test for subjects emphasizing structural processes. Evidence that familiarity has a direct effect on the perceptual organization of the fragmented figures 
(the one-stage model) would be obtained if structural processors exhibited a significant interaction between the effects of familiarity and fragmentation. Evidence that the effects of familiarity occur subsequent to the perceptual organization of the fragmented figures (the two-stage model) would be obtained if the effects of familiarity and fragmentation were additive for structural processors.

\section{METHOD}

\section{Stimuli}

The stimuli were pairs of black uppercase alphabet letters (24 pt. Copperplate-Gothic Heavy Prestype) presented on a white background. Since familiarity was manipulated by presenting the letters in either their normal orientation or rotated $180^{\circ}$ into an unfamiliar orientation, letters that were invariant under $180^{\circ}$ rotation were not used. The letters were arranged horizontally, and subtended a visual angle of $2.4^{\circ}$, at their farthest points, when presented in a two-channel Scientific Prototype tachistoscope.

The fragmented figures were constructed by deleting small sections of alphabet letters. Each of the 12 letters appearing in the experiment was fragmented in three different ways. One fragmentation occurred in a "same" pair; the other two fragmentations occurred in "different" pairs, which always involved different letters, not different fragmentations of the same letter. Each fragmentation appeared once in the familiar orientation and once in the unfamiliar orientation of a letter. As a result, the deleted information was identical in both orientations and the opportunity for recognizing particular fragmentations was minimized.

For half the stimuli, the two letters in a pair were the "same"; for the other half they were "different." Whether a pair of letters constituted a "same" or "different" stimulus, both letters were intact or both were fragmented. When a "same" pair was composed of fragmented letters, they were each fragmented in the same manner so that they constituted a physically identical match. Examples of the stimuli used in this experiment are presented in Figure 1.

\section{Design}

The stimuli were assigned to experimental conditions according to the orthogonal combination of three experimental variables: same-different, intact-fragmented, and normal-rotated, with 12 stimuli falling within each combination. The resultant total of

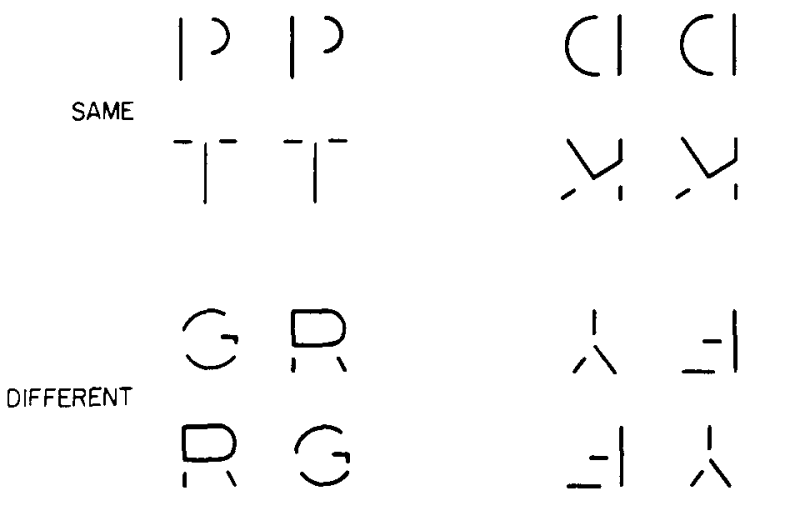

NORMAL ORIENTATION ROTATED ORIENTATION

Figure 1. Examples of fragmented stimuli used in this experiment.
Table 1

Mean "Same" Reaction Time (in Milliseconds) and Percentage of Commission Errors*

\begin{tabular}{lrr} 
& Intact & Fragmented \\
\hline Normal (N) & $398(1.5)$ & $457(.5)$ \\
Rotated (R) & $417(2.0)$ & $496(1.0)$ \\
$(\mathrm{R}-\mathrm{N})$ & $19(.5)$ & $39(.5)$ \\
\hline
\end{tabular}

*Indicated in parentheses

96 stimuli were presented in a randomly mixed sequence that was different for each subject. The experimental trials were preceded by demonstrations of intact and fragmented letters and 12 practice trials with a mixed sequence of intact and fragmented numerals.

\section{Procedure}

A small fixation dot was present before the start of each trial. The stimuli were then presented for a period of $3 \mathrm{sec}$, unless the subjects responded, whereupon the display was terminated. Subjects were instructed to respond, by pressing a button with the thumb of the preferred hand, whenever the two letters were the "same." When the letters were different, they were not to respond in any way. ${ }^{2}$ Subjects were instructed to respond as quickly as possible, with the restraint of keeping their errors to a minimum. The reaction time from the onset of the stimulus display was the dependent measure of primary concern.

Subjects

Sixteen male undergraduate students at Johns Hopkins University voluntarily participated in this experiment. They were paid $\$ 2$ for an experimental session lasting about $20 \mathrm{~min}$.

\section{RESULTS}

The mean "same" reaction times and errors of commission are presented in Table 1. Analysis of variance on the "same" reaction times indicated that the effect of orientation (normal vs. rotated), $F(1,15)=18.64$, the effect of fragmentation (intact vs. fragmented letters), $F(1,15)=73.06$, and the interaction between orientation and fragmentation, $F(1,15)=14.81$, were all significant, $p<.005$. Errors of commission, which averaged $1.3 \%$, were evenly distributed (there was only one error of omission). This error rate, however, was too small to draw any firm conclusions concerning differential speed-accuracy criteria among the various experimental conditions.

Of further concern was the possibility of individual differences in processing underlying "same" responses. Based upon previous studies (Hock, 1973; Hock, Gordon, \& Corcoran, 1976; Hock, Gordon, \& Gold, 1975; Hock, Gordon, \& Marcus, 1974; Hock \& Ross, 1975), individual differences in processing were inferred from the effect, on each subject's "same" responses, of rotating the intact letters into an unfamiliar orientation. Emphasis on structural processes was inferred for subjects with large rotation effects for the intact letters; emphasis on analytic processes was inferred for subjects with small rotation effects for the intact letters. ${ }^{3}$

Interaction contrasts (Sternberg, 1969) were also 
computed for each subject. This was done by determining the effect of fragmentation (fragmented minus intact) for the rotated letters, and subtracting from it the effect of fragmentation for the normally oriented letters. A positive interaction contrast would indicate that the size of the fragmentation effect was smaller for pairs of normal letters than for pairs of letters rotated into an unfamiliar orientation.

The interaction contrasts and rotation effects (for intact letters) of each subject are plotted on the scattergram presented in Figure 2. The correlation coefficient computed for this scattergram was significant, $r=0.71, p<.01 .^{4}$ An examination of the scattergram indicated that negative interaction contrasts were obtained only for subjects with small or negative rotation effects for the intact letters (the latter indicating analytic processing). Subjects with large rotation effects for the intact letters (indicating structural processing) all had positive interaction contrasts. The significant interaction between orientation and fragmentation could therefore be attributed to the data from subjects who emphasized a structural mode of processing. Separate analyses of variance were performed for the eight subjects with the largest rotation effects for the intact letters (structural processors) and the eight subjects with the smallest rotation effects for the intact letters (analytic processors). A significant interaction was obtained for the structural group, $F(1,7)=64.50$, $p<.005$, but not for the analytic group, $F(1,7)<1.0$. The mean "same" reaction times for these two groups of subjects are presented in Table 2.

\section{DISCUSSION}

The significant interaction between orientation and fragmentation supported the hypothesis that

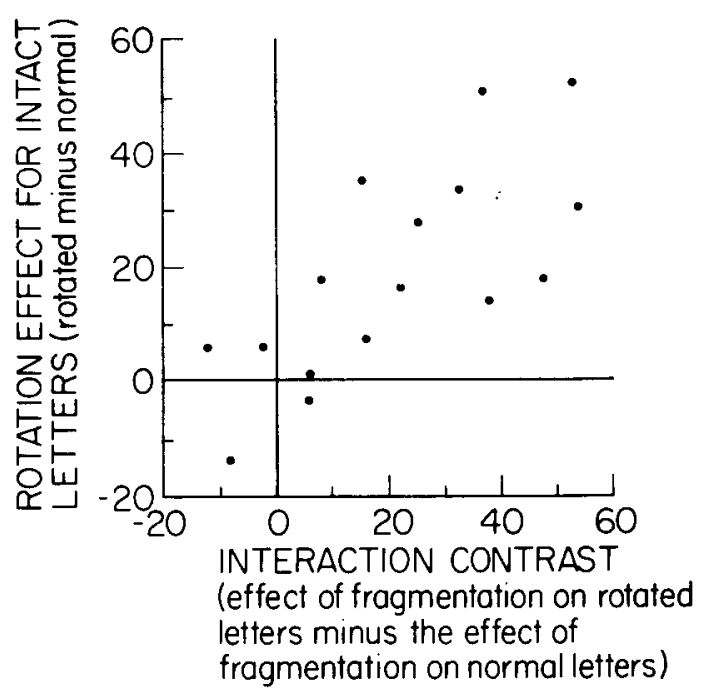

Figure 2. Scattergram for "same" responses. Each point represents the data for one subject.
Table 2

Mean "Same" Reaction (in Milliseconds), with Subjects Divided into Two Groups According to Individual Differences in Processing

\begin{tabular}{lcc}
\hline & Intact & Fragmented \\
\hline & Eight & Structural \\
Normal (N) & 404 & 463 \\
Rotated (R) & 437 & 529 \\
(R-N) & 33 & 66 \\
& Eight Analytic Processors \\
Normal (N) & 392 & 452 \\
Rotated (R) & 396 & 464 \\
(R-N) & 4 & 12 \\
\hline
\end{tabular}

these two variables affect a common stage of processing. Obtaining this interaction for subjects emphasizing structural processes was important for two reasons. First, it implied that the common stage affected by orientation and fragmentation involved structural/organizational processing rather than analytic processing. Second, previous studies (Hock, 1973; Hock \& Ross, 1975) have shown that for structural processors the manipulation of orientation (normal vs. rotated) and the direct manipulation of familiarity (familiar vs. unfamiliar) have equivalent effects on "same" reaction time. This, together with Rock and Heimer's (1957) evidence that rotation has a substantial effect on the recognition of fragmented figures, made it reasonable to conclude that rotation constituted a meaningful manipulation of familiarity. The experimental results were therefore consistent with Leeper's (1935) assertion that familiarity can have a direct effect on the structural/organizational processing of fragmented figures.

The results were inconsistent with the Gestalt contention (Koffka, 1935; Zuckerman \& Rock, 1957) that familiarity does not affect organizational processes. Support for this contention would have been obtained if the effects of orientation and fragmentation had been additive. This result would have indicated that the two variables affected different stages of processing.

The significant interaction between orientation and fragmentation is readily explained in terms of Neisser's (1967) theory, from which it could be argued that the synthesis of the fragmented information into an organized unit is facilitated by schematic information in memory. The finding that the effect of fragmentation was smaller for normally oriented than for rotated letters could then be attributed to the failure of memorial schemes to influence synthetic/organizational processes when the letters were in an unfamiliar orientation.

Before concluding, it should be noted that the results of this experiment cannot be generalized to all stimuli or to all levels of fragmentation of the stimuli. It is obvious that if too little information 
were deleted from a stimulus there would be minimal effect of fragmentation on perceptual processing. Similarly, if too much information were deleted it could become impossible for an appropriate memory trace to be retrieved. Most important, perhaps, is the kind of information that is deleted. In the present experiment, our deletion procedure focused on the points where two or more lines intersected. There was, however, no a priori reason other than our own intuition that this was the "best" way to fragment the letters.

It is conceivable that future research can elaborate on the nature of structural/organizational processes by the systematic deletion of information from a figure. That is, the theoretical structural schemes underlying the perception of a letter could be investigated by deleting information that is critical to the scheme. While it is early to speculate on the nature of these schemes, the spatial nature of structural processing suggests that they may involve a specification of the spatial relations between the parts of a figure as well as the intersections in the figure that represent critical foci for structural/ organizational processes. These spatial specifications may serve as a kind of blueprint that enables Gestalttype structural rules to influence perceptual organization.

\section{REFERENCE NOTES}

1. Hock. H. S., Whitehurst. R.. \& Throckmorton, B. Alternative modes of processing and the retention of previously seen stimuli. In preparation.

\section{REFERENCES}

Bruner, J. S. On perceptual readiness. Psychological Review. 1957, 64, 123-152.

Egeth, H. \& Blecker. D. Differential eftects of familiarity on judgments of sameness and difference. Perception \& Psychophysics, 1971, 9. 321-326.

Hock, H. S. The effects of stimulus structure and familiarity on same-different comparison. Perception \& Psychophysics. 1973. 14. 413-420.

Hock, H. S., Gordon, G. P., \& Corcoran, S. K. Alternative processes in the identification of familiar pictures. Memory \& Cognition. 1976. 4, 265-271.

Hock. H. S., Gordon, G. P. \& Gold, L. Individual differences in the verbal coding of familiar visual stimuli. Memory \& Cognition, 1975. 3. 257-262.

Hock. H. S., Gordon, G. P., \& Marcus. N. Individual differences in the detection of embedded figures. Perception \& Psychophysics, 1974, 15, 47-52.

Hock, H. S., \& Ross, K. The effect of familiarity on rotational transformation. Perception \& Psychophysics, 1975, 18, 15-20.

KOFFKA, K. Principles of Gestalt psychology. New York: Harcourt, Brace and World, 1935.

LEEPER, R. W. A study of a neglected portion of the field of learning - the development of sensory organization. Joumal of Genetic Psychology. 1935, 46, 41-75.

NEISSER, U. Cognitive psychology. New York: Appleton-CenturyCrofts. 1967.

Rock, I., \& Hermer, W. The effect of retinal and phenomenal orientation on the perception of form. American Joumal of Psychology, 1957, 70, 493-511.

STERnBERG. S. The discovery of processing stages: Extensions of Donder's method. Acta Psychologica, 1969, 30, 276-315.

ZuCKerman, C. R., \& Rock, I. A reappraisal of the roles of past experience and innate organizing processes in visual perception. Psychological Bulletin, 1957. 54, 269-296.

\section{NOTES}

1. This explanation for the immediate recognition of previously seen fragmented figures was originally offered by Zuckerman and Rock (1957). They argued that subjects retain memory traces of both the fragmented and reorganized versions of a previously seen fragmented figure. When they see the fragmented figure again, they can immediately retrieve the associated "correct" reorganization that was achieved during the initial examination of the fragmented figure.

2. Only "same" responses were obtained since Egeth and Blecker (1971), Hock (1973), and others have found that rotating familiar stimuli into an unfamiliar orientation affects "same," but not "different," reaction time.

3 . The effect of rotation on "same" reaction time therefore served as both a measure of familiarity and of individual differences. There was no confounding involved here. Previous studies (Hock, 1973; Hock \& Ross, 1975) have shown that the "same" responses of both structural and analytic processors are affected by familiarity (familiar vs. unfamiliar stimuli). However, the effect of familiarity is invariant under rotation (familiar vs. rotated-familiar) for analytic processors, but not structural processors.

4. Although the two variables entering into the correlation were not independent, the effect of this was to suppress rather than enhance the obtained correlation.

(Received for publication April 5, 1976; revision accepted July 30,1976 .) 